\title{
El ius Latii y la integración jurídica de Occidente. Latinización vs. romanización
}

\author{
The ius Latii and the legal integration of the Western Roman \\ Empire. Latinization vs. Romanization
}

DAVID ESPINOSA ESPINOSA

\begin{abstract}
RESUMEN
En base a los problemas de interpretación histórica que generan las comunidades de derecho latino, $y$ apoyados en el análisis onomástico de la población residente en dichas comunidades, proponemos la idoneidad del empleo del término latinización, frente al tradicional concepto de romanización, para referirnos al proceso de integración jurídica de las poblaciones indígenas de Occidente en época romana.
\end{abstract}

PALABRAS CLAVE:

Derecho latino, derecho romano, latinización, romanización, onomástica, epigrafía, Hispania, Occidente.

\begin{abstract}
Considering the problems of historical interpretation that generate the communities of Latin right, and drawing on the onomastic analysis of the resident population in those communities, we propose the suitability of the employment of the term latinization, as opposed to the traditional concept of romanization, to refer to the process of juridical integration of indigenous populations in the Western Roman Empire.
\end{abstract}

\section{KEYWORDS:}

Latin right, Roman right, latinization, romanization, onomastic, epigraphy, Hispania, Western Roman Empire.

\section{ROMA, CIUDAD ABIERTA E INTEGRADORA}

El mundo antiguo mediterráneo, y la Península lbérica en su contexto, estaban constituidos por un vasto mosaico de pueblos y culturas que, en mayor o menor medida, en algún momento de su historia, entraron en contacto unos con otros.

* Departamento de Historia Antigua, Universidad Complutense de Madrid (C/Profesor Aranguren s/n. 28040 Madrid). E-mail: davidespinosa@ghis.ucm.es.

Artículo basado en la comunicación leída el 25 de Mayo de 2009, en la VIII edición del Encuentro de Jóvenes Investigadores de Historia Antigua de la Universidad Complutense de Madrid. 
Éste fue el caso de Roma, que al ritmo de su expansión, primero en Italia, y poco después por el resto de la cuenca mediterránea, fue hallando en su avance infinidad de comunidades en diferente grado de progreso.

Roma, considerada por excelencia una ciudad-estado abierta e integradora (Sherwin-White 1973: 5-7), desarrolló un complejo entramado jurídico-administrativo con el objetivo de ampliar su territorio y cuerpo cívico. El derecho de asilo, la tribus, el ius migrandi, la civitas sine suffragio, la colonia romana y latina, y el municipio romano y latino, constituyeron la infraestructura empleada por Roma en una política exterior de integración jurídica (Salmon 1969; Sherwin-White 1973; Humbert 1978; Luraschi 1979) fundamentada en la sympoliteia (Pascual 2007: 167186), la isopoliteia (Pascual 2007: 167-186; Humbert 1978: 85-143), el hospitium publicum (Santiago y Gardeñes 2002: 7-36; Nicols 2001: 99-108; Humbert 1978: 85-143; Balbín 2006: 207-235), la humanitas (Bauman 1999; Hingley 2005; Veyne 1993; Woolf 1998; Nybakken 1939: 396-413) y la libertas (Humbert 1976: 221-242), es decir, en el reconocimiento del extranjero como potencial ciudadano romano y la protección de su persona y sus bienes. Este genuino sistema de gobierno, surgido del deseo de integrar jurídicamente a lo 'no romano', a lo indígena, al 'otro', originó la institucionalización de la diferencia, la asimilación de un sinfín de culturas y la exportación del modus vivendi romano.

Como señalara el orador griego Elio Arístides (Panegírico a Roma 56-60, 6371,102 ), «lo más original y maravilloso (de Roma) es la grandeza de tu ciudadanía. Has dividido a todos los pueblos del Imperio, lo cual significa todo el mundo, en dos clases. Los más civilizados y mejor nacidos en ciudadanos romanos; el resto, está subordinado y sometido. Este Estado es universal, con leyes comunes (nómoi koinoí), magistrados que tratan a los gobernados no como extranjeros sino como a su propio pueblo, y castigan a los funcionarios corruptos. Que ha habido guerras, nadie lo duda, pero para la mayoría de los pueblos son meras leyendas", o el naturalista latino Plinio el Viejo (NH3.39), nec ignoro ingrati ac segnis animi existimari posse merito, si obiter atque in transcursu ad hunc modum dicatur terra omnium terrarum alumna eadem et parens, numine deumelecta quae caelum ipsum clarius faceret, sparsa congregaret imperia ritusque molliret et tot populorum discordes ferasque linguas sermonis commercio contraheret ad conloquia et humanitatem homini daret breviterque una cunctarum gentium in toto orbe patria fieret, el modelo jurídico de sometimiento utilizado por el Estado romano no tuvo como objeto la esclavización de las poblaciones, sino que optó por generar sociedades políticas de ciudadanos romanos y peregrinos sometidos a su dominio con el objetivo de crear una communis patria (García Fernández 2007: 311-321).

Augusto en su testamento político - Res Gestae 3.2: externas gentes, quibus tuto ignosci potuit, conservare quam excidere malui-, o Virgilio en su célebre epopeya latina -Eneida 6.851-53: «tú, Romano, acuérdate de regir a los pueblos con tu imperio/(éstas serán tus artes), de imponer las leyes de la paz,/de perdonar a los vencidos y domeñar a los soberbios»-, resumieron una vez más esta misma idea, la clara voluntad de dominio universal por parte de Roma y su vocación integradora respecto a las comunidades sometidas. 
¿Cuáles fueron las causas de este proceso integrador? Como explicara Pierangelo Catalano en un magnífico artículo sobre los aspectos espaciales del sistema jurídico-religioso romano, la razón última de este espíritu reside en la construcción, por parte de Roma, de un sistema ideológico y jurídico universal por el cual el extranjero formaba parte de su esencia a través de su incorporación a la ciudadanía romana, incorporación que comportaba dioses, hombres y territorios (Catalano 1978: 523): Il presupposto di questa volontà giuridica e politica che tende ad una societá universale è, torno a sottolineare, un'intuizione dello 'spazio', la quale coinvolge dii, homine, loca.

Pero además, como pusieran de manifiesto ya en la Antigüedad diversos pensadores grecorromanos, Roma estaba imbuida en un proceso de perfeccionamiento constante de su ciudadanía, consistente en el deseo de recuperar la energía y los valores primordiales romanos, corruptos por los vicios de la civilización, presentes en los pueblos recientemente sometidos. Según el pensamiento romano, era posible el acceso del bárbaro a la humanitas, a través de la civitas, como, por el contrario, era también posible la caída del civilizado en un estado de barbarie. Roma, por lo tanto, tuvo el convencimiento de la necesidad de integrar a las comunidades indígenas para regenerar su cuerpo cívico y extraer, de éste, una nueva virtus. En este sentido, Séneca, uno de los principales representantes del pensamiento estoico, propugnaba la vuelta al romano de antaño, frugal, moderado, para recuperar, desde la razón y la cultura latina, la esencia de Roma (De ira 2.11.14), y construir un 'Estado universal', u orbis. En definitiva, no era posible una civilización equilibrada sin la presencia, bajo los parámetros de la vida ciudadana, de la energía primordial latente entre los bárbaros (Gómez Santa Cruz 2007: 117-40).

\section{PILARES DEL PROCESO DE INTEGRACIÓN JURÍDICA}

Roma extendió su imperium en base a dos mecanismos estratégicos: el aprovechamiento de las condiciones preexistentes en cada territorio conquistado, y la aplicación, en éstos, de esquemas de amplia difusión que se particularizaban en el momento de su aplicación.

Respecto al primero de ellos, es una constante en la política romana, un signo distintivo, la posibilidad de aprovechar las condiciones existentes, adaptándolas eficazmente a sus necesidades. Esta es también la conclusión a la que llega M. Dondin-Payre (1999: 225), quien afirma que la fuerza de la organización del Imperio romano radicó, precisamente, en su facultad de adaptación a las condiciones locales en el marco de cuadros generales, y no en la imposición de modos de gobierno idénticos. En este sentido, la existencia de núcleos urbanos prerromanos, de oppida, o de organizaciones suprafamiliares, fue un valioso apoyo, pero no indispensable, para que la integración jurídica romana fuera posible.

En cuanto al segundo, Roma implantó decididamente el modelo de ciudad, de civitas, y procedió a la progresiva y selectiva extensión de sus derechos ciudadanos, de sus iura, materializado en la deducción de colonias y en la promoción de 
las comunidades locales al status de municipio. Ciudad y ciudadanía llevaron a la integración, estabilidad y unidad del Imperio, un Imperio fraguado en el yunque del derecho y de la vida urbana. Roma podría haber preservado celosamente su ciudadanía, dejando a las comunidades sometidas en un estado permanente de sujeción. Pero, sorprendentemente, en un proceso sin precedentes, concedió sus derechos a las comunidades indígenas, regulando de este modo la convivencia y su relación con ellas.

Roma instituyó numerosas ciudades a lo largo de todo el Mediterráneo, pero, realmente, extendió una única idea de ciudad: la civitas. Marco estructural desde el que operaba el Estado romano, comprendía dos dimensiones fundamentales, la urbana, urbs, concreción física de la comunidad humana en el espacio, y la sociopolítica, civitas, conjunto de ciudadanos unidos por una misma constitución y dotados de un conjunto de derechos (iura) y obligaciones (munera). En base a ella, podemos entender cómo Roma aprovechaba, asimilaba, transformaba y ampliaba la compleja realidad preexistente para integrar los territorios conquistados.

El Estado romano creó, fundamentalmente, dos niveles de ciudadanía: el Romano, que comportaba el pleno disfrute de los privilegios inherentes a la ciudadanía romana, y el Latino, que garantizaba la aplicación de los principios fundamentales del ius civile romano, y ajustaba a derecho los usos y costumbres de las comunidades indígenas.

\subsection{Naturaleza jurídica y desarrollo histórico de la Latinidad}

La latinidad fue un expediente jurídico complejo, empleado por Roma, para favorecer la integración de comunidades indígenas en los modos de vida romanos, sin pasar, previamente, por una concesión de ciudadanía romana. Forjado al ritmo de las relaciones entre Roma y los itálicos, el Latium (Asconio, In Pis. 3c; Strab. 4.1.12; Gayo, Inst. 1.95; App. 2.26; Tac. Ann. 15.32, Plin. NH3.30, 91, 135; Cic. Ad Att. 14.12.1) comprendía un conjunto de derechos civiles que actuaban como marco legal de regulación de sus relaciones con Roma: ius conubii, ius commercii e ius suffragii (Humbert 1978: 23-26, 130-43; Sherwin-White 1973: 98-116, 375-79). De éstos, conubium y commercium eran los más importantes, pues cubrían la esfera de las relaciones sociales fuera de la comunidad cívica de origen. Además, ofrecían la posibilidad de acceder a la ciudadanía romana (García Fernández 2007: 311-21), en un primer momento a través del ius migrandi (Sherwin-White 1972: 108-16), y posteriormente mediante el ejercicio del ius adicispendae civitatem per magistratum (Tibiletti 1953: 46-63; Piper 1988: 61; García Fernández 2001: 150-51), es decir, tras el desempeño de una magistratura en la comunidad promocionada con derecho latino. Obtener la ciudadanía per migrationem et census no pertenece al mismo nivel de pensamiento político que el proceso per magistratum o per honorem, ya que en el primero el latino se ve obligado a cambiar de domicilio y en el segundo disfruta de su nuevo status ciudadano en su vieja patria. Por ello, conubium y commercium resultan fundamentales, pues evitan su aislamiento y extinción jurídica. 
Siguiendo un esquema cronológico tripartito, tras ser empleado en el Lacio por los estados miembros del Foedus Cassianum (493 a.C.), siglos V y IV a.C., al calor de los acontecimientos militares, y de la mano de los grandes prohombres de la República, comenzó a ser difundido por Occidente siguiendo las pautas del procedimiento colonial (Humbert 1978: 151-250; Sherwin-White 1972: 96-118), originando el conocido conjunto de colonias latinas deducidas en Italia y Galia Cisalpina, y el controvertido grupo fundado en ámbito provincial, cuyos ejemplos más claros lo constituyen Carteia, en Hispania (Liv. 43.3.1-4), y Aquae Sextiae, en Galia Narbonense (Roman 1987: 185-90). Fundamental en su extensión fue el hecho de que superara sus limitaciones geográficas iniciales para, concibiéndose en un sentido sociopolítico, convertirse en una fórmula de integración puramente jurídica. El estatuto latino se configuró, de este modo, como un nivel de ciudadanía romana, desde la cual se origina y con la cual coincide parcialmente, caracterizado por una disminución de los derechos y privilegios que comportaba. Pero a partir del año 89 a.C., en plena convulsión provocada por la Guerra de los Aliados, y como consecuencia de una lex Pompeia de Transpadanis (Asconio, In Pis. 3c; Luraschi 1979: 331), la latinidad sufrió una profunda mutación (García Fernández 1999: 279-87; 2001: 16-22). Extinguidas las antiguas colonias latinas itálicas, mediante su transformación en municipios romanos de la mano de la Lex lulia de Civitate, el nuevo expediente latinizador, de carácter municipalizante (Luraschi 1979: 165-66; 1983: 265), posibilitaría la promoción de las comunidades peregrinas sin necesidad de reordenamiento constitucional alguno ni de reorganización territorial inmediata, resultando económico para el Estado romano y posibilitando la pervivencia de las estructuras organizativas indígenas, ajustadas a derecho.

El siguiente territorio de aplicación de esta nueva latinidad será la Galia Transalpina, donde el expediente transpadano aparece dotado de una mayor uniformidad, si bien las características básicas seguirían siendo las mismas: concesión de ius Latii, ausencia de deducción y adquisición de titulatura colonial.

Mientras que la regularización y desarrollo del expediente transpadano se produjo en la Galia Narbonense, el ajuste de sus características constitucionales a una titulatura adecuada parece haber tenido lugar en Hispania (García Fernández 2000: 73-83). Después del episodio narbonense, no vuelve a documentarse titulación colonial latina, siendo la municipal la presente en comunidades como Ercauica, Cascantum, Lucentum o Saetabis, cuya información se fecha en época augustea. Este grupo de comunidades designadas por Plinio como oppida de antiguo Lacio, que remiten a una latinización republicana hispana, mal conocida y compleja, presentan cierto tono de homogeneidad que las vincula a la labor municipalizadora y reorganizadora realizada por Augusto. Salvo alguna mención aislada a la tribus Sergia, asignada por César a sus promociones, prácticamente todas las comunidades de Lacio antiguo están adscritas a la tribu Galeria (Stylow 1995; Wiegels 1985), lo que apunta a un programa global de concesión y de reorganización de las comunidades latinas hispanas promovido por Augusto.

Por la acción de dicho privilegio, convivían en una misma comunidad individuos con dos niveles diferentes de ciudadanía (García Fernández 1998: 209-21; 2001 : 
125-80), la latina (Gardner 1993: 188-90), a la que se refieren algunos pasajes de la legislación flavia a través de quienes la disfrutan (Lex Irnitana, cap. 28 y 72; Lex Malacitana, cap. 53), y la romana, presente en la documentación epigráfica. El resultado, comunidades mixtas regidas por un modus vivendi regulado por el ius civile romano.

\section{LATINIZACIÓN VS. ROMANIZACIÓN}

En este novedoso escenario interpretativo, proponemos el uso del versátil concepto de latinización frente al tradicional y controvertido de romanización. Según la historiografía, este último constituiría un intenso proceso de reconfiguración de las condiciones de existencia de las comunidades indígenas próximo a un fenómeno de asimilación cultural, reduciéndose el papel de estas comunidades al de meros espectadores.

Pero, como se viene apuntando en las últimas décadas, lejos queda de esta noción la realidad histórica de la mayoría de las comunidades encuadradas en las estructuras organizativas del Imperio, debiéndose analizar la integración desde un proceso de intereses mutuos y de adopción formal, por parte de estas, de usos, formas y costumbres de la cultura y sociedad romanas. Debe tenerse presente, además, que el concepto de romanización presenta, al menos, tres vertientes distintas: jurídica, entendida como la expansión de los derechos de ciudadanía romana, política-institucional, caracterizada por la adopción de las instituciones cívicas y religiosas romanas, y cultural, que constituía la introducción de prácticas, hábitos y modas romanas. Según esto, mientras que algunas comunidades habrían adquirido un grado de romanización absoluto, las menos numerosas, el resto permanecería invariable salvo por la introducción de algunos elementos de filiación romana.

Por si fuera poco lo anterior, hay que tener en cuanta que, hasta la concesión de ciudadanía romana por el emperador Caracalla en el año 212 d.C. a todo el orbe romano, más de tres cuartas partes de la población occidental del Imperio era de condición latina y no romana. Por lo tanto, pensamos que es preferible, al menos en Occidente, hablar de latinización y no de romanización. La romanización, en este sentido, no habría sido sino un espeso barniz de romanidad que permitió la pervivencia de las identidades indígenas. Coyunturas desfavorables para el Imperio, como la crisis del siglo IV a.C., facilitaron su afloramiento y revitalización. Si la romanización hubiera sido plena y efectiva, este hecho no hubiera sido posible.

\subsection{Análisis onomástico de la población de las comunidades latinas}

Con el propósito de demostrar la idoneidad del empleo del término latinización, resulta de gran valor e interés su aplicación en el análisis de la estructura onomástica de la población residente en las comunidades promocionadas con derecho latino, pues presentan numerosos problemas de interpretación histórica. 
En este tipo de comunidades, se constata el uso generalizado de estructuras onomásticas romanas - dua o tria nomina - e indígenas —nombre único más filiación-. Si utilizáramos el modelo explicativo clásico, dichas estructuras corresponderían a ciudadanos romanos y peregrinos, haciendo desaparecer toda la población latina del Occidente romano que habitaba en los denominados oppida Latina plinianos:

Edeta (Liria, Valencia):

$$
\begin{gathered}
\text { HEp 2, 703: } \\
\text { M(arco) CORNELIO } \\
\text { M(arci) F(ilio) GAL(eria tribu) } \\
\text { NIGRINO } \\
\text { FILIO }
\end{gathered}
$$

A Marco Cornelio Nigrino, hijo de Marco, de la tribus Galeria, hijo.

CIL II, 3794:

SERANVS TAN

NEGISCERRIS

F(ilius) OTOBESANV[s]

Serano, hijo de Tannegiscerris, Otobesano.

Ercavica (Cañaveruelas, Cuenca):

CIL II, 4203:

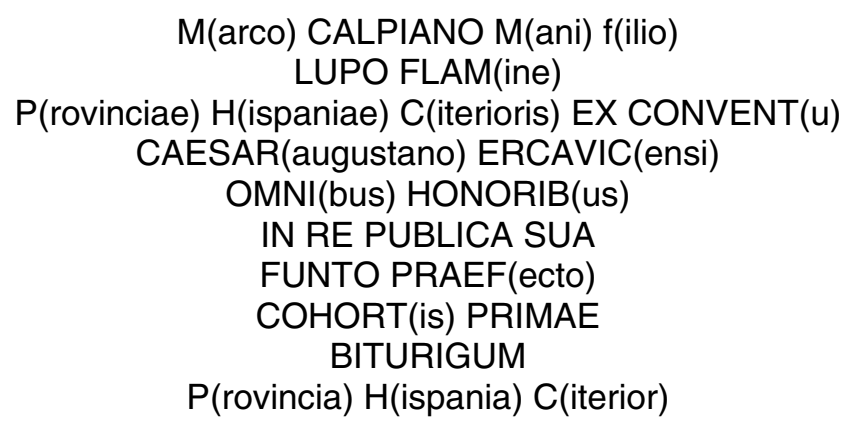

La Provincia Hispania Citerior a Marco Calpiano Lupo, hijo de Mano, flamen de la Provincia Hispania Citerior, del Conventus Caesaraugustano, Ercavicense, desempeñó todos los honores en su comunidad, prefecto de la primera cohorte Biturigum.

CIL II, 3166:

PROCULO

PELLICO

FILI 
$\mathrm{FACl}($ endum)

[curaverunt]

A Próculo, hijo de Pellico, mandaron hacerlo.

Pero, además, si no consideramos la existencia de latinos detrás de estas estructuras onomásticas, corremos el riesgo de admitir situaciones ilícitas o poco corrientes:

Uso de dua o tria nomina por parte de poblaciones peregrinas: cuando Suetonio (Claudio 25) nos informa de la prohibición que tenían los peregrinos de usar nomina romanos: Peregrinae condicionis homines vetuit usurpare Romana nomina dum taxat gentilicia. Civitatem R. usurpantes in campo Esquilino securi percussit. provincias Achaiam et Macedoniam, quas Tiberius ad curam suam transtulerat, senatui reddidit; cuando el emperador Claudio censuró a la tribu alpina de los Anauni la usurpación de la ciudadanía romana en su edicto de Civitate Anaunorum (CIL V, 5050; 46 d.C.): Quod ad condicionem Anaunorum et Tulliassium et Sindunorum pertinet, quorum partem delator adtributam Tridentinis, partem ne adtributam quidem arguisse dicitur, tametsi animaduerto non nimium firmam id genus hominum habere ciuitatis Romanae originem, tamen cum longa usurpatione in possessionem eius fuisse dicatur et ita premix tum cum Tridentinis, ut diduci ab is sine graui splendidi municipi iniuria non possit, patior eos in eo iure, in quo esse se existimauerunt, permanere beneficio meo, eo quidem libentius, quod plerique ex eo genere hominum etiam militare in praetorio meo dicuntur, quidam uero ordines quoque duxisse, nonnulli allecti in decurias Romae res iudicare: cuando Roma acusó públicamente al gaditano Lucio Cornelio Balbo y al poeta griego Aulo Licinio Arquías de usurpar la ciudadanía (Cic. Pro Balbo; Pro Archias).

Presencia de un numeroso grupo de población peregrina en el interior de comunidades promocionadas, sean colonias o municipios, con derecho latino: como en Salacia (Alcaçer do Sal, Setúbal), donde un individuo con onomástica peregrina, Vicanus, realiza una dedicatoria al emperador Augusto:

CIL II, 5182:

IMP(eratori) CAESARI DIVI F(ilio) AUGVSTO

PONTIFICI MAXIMO CO(n)S(uli) XII

TRIB(unicia) POTESTATE XVIIII

VICANVS BOUTI F(ilius)

SACRVM

Consagrado al emperador Augusto, hijo del divino César, Pontífice Máximo, en su XII consulado, en su XVIIII potestad tribunicia. Vicano, hijo de Bouto; como en Edeta (Liria, Valencia), donde Ambatus cumplió su voto a una divinidad desconocida:

CIL II, 3787:

AMBATUS

EX VOTO 
A ..., Ambato cumplió su voto; como en Ercavica (Cañaveruelas, Cuenca), donde fue enterrado Caecilius, hijo de Senicio:

$A E$ 1982, 617:

\author{
CAIICILIVS \\ SIINICIONIS F(ilius) \\ $\mathrm{H}$ (ic) S(itus) IIST
}

Cecilio, hijo de Senicio. Aquí está enterrado.

Existencia de uniones matrimoniales entre un ciudadano romano y una mujer de condición peregrina, cuyos descendientes heredan la ciudadanía romana: uniones y transmisiones patronímicas ilegítimas siempre y cuando Roma no concediese a la comunidad en la que residían el ius conubii.

CIL XII, 1133:

\author{
NOVELLIA NOVANI F(ilia) PATERNA SIBI \\ $P$ (ublio) NOVELLIO NOVANO PATRI SABI \\ NAE LENAEI F(ilia) MATRI, P(ublio) NOVELLIO \\ VASTO L(UCIO) NOVELLIO SABINO FRATRI \\ BUS Q(uinto) VERATIO [VE]RO SEX(to) VERATIO \\ PATERNO NEPOTIBUS
}

Novelia Paterna, hija de Novano, para si, para su padre Publio Novelio Novano, su madre Sabina Lanae, sus hermanos Publio Novelio Vasto y Lucio Novelio Sabino, sus nietos Quinto Veratio Vero y Sexto Veratio Paterno.

La flexibilidad onomástica, por tanto, es una característica propia de las comunidades latinas, correspondiendo la denominación de los latinos, como apuntara Alföldy en 1966, a las construcciones onomásticas de peregrinos o romanos. Es por todo ello que debemos reconsiderar, revisar y redefinir los postulados y las bases a partir de las cuales construimos el discurso histórico relativo a las fases de conquista e integración jurídica de Occidente, valorando la complejidad y variabilidad de situaciones que introduce la Latinidad, uniformizadas por la aparente romanización.

Para concluir, quisiera hacer mía una reflexión de la Prof. García Fernández (2001) a cerca de la importancia que tuvo el derecho latino en la política exterior de Roma. Según ésta, "el ius Latii se diseñó de tal manera que permitía la absorción de todo tipo de identidades y tradiciones culturales en el seno del Estado romano, actuando, a la vez, de filtro, para que únicamente los individuos más cualificados pudiesen acceder a la ciudadanía romana. Sin instrumentos de esta naturaleza, la romanización de lo que hoy constituye Europa occidental no hubiera sido posible. Es por esta razón que cualquier intento de solución que se ensaye en relación a la integración y romanización de los territorios conquistados por Roma no puede pasar por alto el episodio latinizador, puesto que la condición latina, que no la romana, fue el vehículo que con preferencia empleó Roma para integrar a Occidente, y especialmente a las provincias hispanas». 


\section{BIBLIOGRAFÍA}

ALFÖLDY, G. (1966), «Notes sur la relation entre le droit de cité et la nomenclatura dans l'Empire Romain», Latomus 25, 37-57.

BALBÍN CHAMORRO, P. (2006), «lus hospitii y ius civitatis», Gerión 24.1, 207-235.

BAUMAN, R. A. (1999), Human Rights in Ancient Rome, London.

CATALANO, P. (1978), «Aspetti spaziali del sistema giuridico-religioso romano. Mundus, templum, urbs, ager, Latium, Italia«, ANRW II.16.1, 440-553.

DONDIN-PAYRE, M. (1999), «Magistratures et administration municipale dans les Trois Gaules», en Dondin-Payre, M. y Raepsaet-Charlier, M. T. eds., Cités, Municipes, Colonies. Les Processus de Municipalisation en Gaule et en Germanie sous le Haut-Empire Romain, Paris, 127-230.

GARCÍA FERNÁNDEZ, E. (1991), «El ius Latii y los municipia Latina», Studia Historica 9, 29-41.

- (1998), «Características constitucionales del municipio latino», Gerión 16, 209-221.

- (1999), «La lex Pompeia de Transpadanis y el origen del municipio latino», en González, J. ed., Ciudades Privilegiadas en el Occidente Romano, Sevilla, 279-287.

- (2000), «Plinio y los oppida stipendiariae. A propósito de un artículo de Alicia M. ${ }^{a}$ Canto», Gerión 18, 571-591.

- (2001), El Municipio Latino. Origen y Desarrollo Constitucional, Anejos de Gerión 5, Madrid.

- (2007), «Ciudadanía e Imperio», Gerión 25, 311-321.

GARDNER, J. F. (1993), Being a Roman Citizen, Londres.

GÓMEZ SANTA CRUZ, J. (2007), "Ciudadanos y extranjeros en el Mundo Antiguo: segregación e integración. El mito del 'buen salvaje' en el mundo romano», en Mangas, J. y Montero, S. eds., Ciudadanos y Extranjeros en el Mundo Antiguo: Segregación e Integración, Madrid, 111-142.

HINGLEY, R. (2005), Globalizing Roman Culture: Unity, Diversity and Empire, London.

HUMBERT, M. (1976), "Libertas id est civitas: autour d'un conflit négatif de citoyennetés au Ile siglo avant J.-C.», Mélanges de l'école française de Rome 88.1, 221-242.

- (1978), Municipium et Civitas sine Suffragio. L'Organisation de la Conquête jusqu'a la Guerre Sociale, Paris.

LURASCHI, G. (1979), Foedus, Ius Latii, Civitas. Aspetti Constituzionali della Romanizzazione in Transpadana, Padua.

- (1983), «Sulla magistratura nelle colonie latine fittizie (a proposito di Frag. Atestinum II. 1012)», SDHI 49, 261-329.

NICOLS, J. (2001), «Hospitium and political friendship in the Late Republic», en Peachin, M. ed., Aspects of Friendship in the Graeco-Roman World, Portsmouth, 99-108.

NYBAKKEN, O. E. (1939), «Humanitas romana», TAPhA 70, 396-413.

PASCUAL, J. (2007), «La sympoliteia griega en las épocas clásica y helenística», Gerión 25, 167-186.

PIPER, D. J. (1988), «The ius adicispendae civitatis Romanae por magistratum and its effect on Roman-Latin relations», Latomus. 47.1, 59-68.

ROMAN, D. (1987), «Aix-en-Provence et les débuts de la colonisation de droit latin en Gaule du sud", Revue Archéologique de Narbonnaise 20, 185-190.

SALMON, E. T. (1969), Roman Colonization under the Republic, Londres.

SANTIAGO ÁLVAREZ, R. A. y GARDEÑES SANTIAGO, M. (2002), «Interacción de poblaciones en la Antigua Grecia: algunos ejemplos de especial interés para el derecho internacional privado", Faventia 24.1, 7-36.

SHERWIN-WHITE, A. N. (1972), The Roman Citizenship, Oxford.

STYLOW, A. U. (1995), «Apuntes sobre las tribus romanas en Hispania», Veleia 12, 105-123.

TIBILETTI, G. (1953), «La politica delle colonie e delle città latine nella guerra sociale», Rendiconti dell'Istituto Lombardo di Scienze e Lettere (RIL) 86, 45-63. 
El ius Latii y la integración jurídica de Occidente. Latinización vs. romanización

VEYNE, P. (1993), «Humanitas: Romans and non-Romans», en Giardina, A. ed., The Romans, London, 385-415.

WIEGELS, R. (1985), Die Tribusinschriften des römischen Hispanien. Ein Katalog, Berlin. WOOLF, G. (1998), Becoming Roman. The Origins of Provincial Civilization in Gaul, Cambridge. 